\title{
Estrogen therapy increases BDNF expression and improves post-stroke depression in ovariectomy-treated rats
}

\author{
QIAOER SU ${ }^{1}$, YIFAN CHENG ${ }^{2}$, KUNLIN JIN $^{3}$, JIANHUA CHENG $^{2}$, YUANSHAO LIN $^{2}$, \\ ZHENZHEN LIN ${ }^{2}$, LIUQING WANG ${ }^{2}$ and BEI SHAO ${ }^{2}$ \\ Departments of ${ }^{1}$ Pediatrics and ${ }^{2}$ Neurology, The First Affiliated Hospital of Wenzhou Medical University, Wenzhou, \\ Zhejiang 325000, P.R. China; ${ }^{3}$ University of North Texas Health Science Center, Fort Worth, TX 76107, USA
}

Received January 14, 2015; Accepted September 9, 2015

DOI: $10.3892 / \mathrm{etm} .2016 .3531$

\begin{abstract}
The present study investigated the effect of exogenous estrogen on post-stroke depression. Rats were exposed to chronic mild stress following middle cerebral artery occlusion. The occurrence of post-stroke depression was evaluated according to the changes in preference for sucrose and performance in a forced swimming test. Estrogen therapy significantly improved these neurological symptoms, indicating that estrogen is effective in treating post-stroke depression. Increased brain-derived neurotrophic factor (BDNF) expression was reported in the hippocampus of rats that had been treated with estrogen for two weeks, suggesting that BDNF expression may be an important contributor to the improvement of post-stroke depression that is observed following estrogen therapy.
\end{abstract}

\section{Introduction}

Depression is a common complication of stroke, occurring in $\sim 33 \%$ of patients $(1,2)$; the clinical symptoms of this include feelings of guilt, low self-esteem, reduction in food intake, sleep disorders and fatigue. There are between 1.6-2.0 million new stroke patients in China every year and post-stroke depression (PSD) is closely associated with the increased risk of mortality following a stroke (3). PSD negatively impacts upon subsequent rehabilitation; in a previous study by Sinyor et al, PSD patients demonstrated greater functional impairment than patients without depression, also scoring lower on behavioral action and functional status (4). The severity of depression is closely associated with health-associated quality of life and functional recovery in stroke survivors (5). PSD patients demonstrate

Correspondence to: Professor Bei Shao, Department of Neurology, The First Affiliated Hospital of Wenzhou Medical University, 2 Fuxue Road, Wenzhou, Zhejiang 325000, P.R. China

E-mail: suqiaoernancy@163.com; shao_bei@yahoo.com.cn

Key words: estrogen, post-stroke depression, brain-derived neurotrophic factor, animal behavior, hippocampus a negative mood during rehabilitation and achieve poorer outcomes during functional recovery therapy. These patients also have greater difficulty in restoring social activities (6). Several studies have described a higher mortality rate in stroke patients with depression (7), and certain studies have reported that PSD patients using antidepressant drugs demonstrate improved ability to function than those without antidepressant therapy. The treatment of depression has been demonstrated to aid functional recovery (8), meaning that the early diagnosis and effective treatment of PSD are crucial to recovery from a stroke.

Brain-derived neurotrophic factor (BDNF) is a member of the neurotrophin family, which regulates neurogenesis (9), apoptosis (10), the expression level of monoamine transmitters (11), and the function and plasticity of synapses (12). Neurotrophins may be key in the development of depression (13); BDNF, for instance, can activate intracellular mitogen-activated protein kinase/extracellular signal-regulated kinase cascade signaling pathways (14), affecting synaptic plasticity (12) and alleviating the symptoms of depression (15), and BDNF may thus be important in the maintenance of emotional stability. Deletion of the BDNF receptor induces a reduction in neurogenesis and increases anxious behavior (16). Additionally, BDNF expression is associated with 5-hydroxytryptamine receptor expression in the brain, particularly the hippocampus (11), and is hypothesized to treat depression in animals by improving the activity of monoamine transmitters in response to antidepressant drugs (17). BDNF is therefore considered to be a key neurotrophic factor that modulates a depressive mood through its activity in the hippocampus. In clinical practice, serum BDNF is lower in patients with depression, but antidepressant treatment can induce an increase in BDNF levels (18), making serum BDNF a sensitive peripheral marker that is predictive of the severity of depression (19) and of the treatment outcome (20). Concordantly, previous studies have indicated that the concentration of BDNF is lower in PSD patients than in non-PSD patients (21). Prior studies predominantly focused on the association between serum BDNF levels and depression severity. BDNF is expressed in multiple regions of the brain, including the hippocampus, and is involved in brain functionality (22), making BDNF levels a useful metric to predict the severity of depression in PSD patients. 
Estrogen is an effective mood regulator and causes antidepressant-like effects on a depression model in rats (23). Estrogen acts on the central nervous system through its nuclear receptors. There are two well-known types of estrogen receptors (ER): $E R \alpha$ and $E R \beta$. ERs are widely distributed in the brain and ER $\beta$ is abundantly expressed in the rat hippocampus (24). Estrogen implements its antidepressive effects by activating $\operatorname{ER} \beta$ (25). Numerous previous studies have indicated that estrogen and BDNF stimulate neurogenesis in the hippocampus $(9,26)$ and are involved in synaptic modification $(27,28)$ to enhance learning and memory (28-30). Resultantly, the present study investigated the association between estrogen and BDNF in the hippocampus and the hypothesis that BDNF is involved in estrogen-mediated antidepressant effects upon PSD.

\section{Materials and methods}

Animals. Female two-month-old Sprague-Dawley rats, each weighing 230-250 g, were used in all experiments (Wenzhou medical University, Wenzhou, China). The rats were housed under the following conditions: A 12:12-h light-dark cycle, with lights on at 7:00 a.m.; temperature maintained at $21 \pm 2^{\circ} \mathrm{C}$; and the provision of food and water ad libitum. All animal procedures were performed in accordance with the guidelines of the Animal Care and Use Committee of Wenzhou Medical University. All surgical procedures were performed under chloral hydrate anesthesia (21). In order to develop a PSD model, the rats were randomly divided into four groups as follows: The sham/control group, the middle cerebral artery occlusion (MCAO)/control group, the sham/chronic mild stress (CMS) group and the MCAO+CMS group $(n=20)$. Comparison of these groups was used to validate the animal models. The sham/control (control) group and the MCAO+CMS (PSD) group were then randomly divided into matched subgroups for subsequent estrogen treatment, as follows: The vehicle-treated control group, the estradiol (E2)-treated control group, the vehicle-treated PSD group and the E2-treated PSD group $(n=10)$. Comparison of the four groups was used to evaluate the effect of estrogen on PSD. The entire experiment lasted for six weeks (Fig. 1).

Ovariectomy. All rats were subjected to an ovariectomy prior to grouping. All experimental procedures were approved by the ethics committee of Wenzhou Medical University. Under chloral hydrate anesthesia $(3.2 \mathrm{mg} / \mathrm{kg})$, rats were kept in a supine position on the operating table. Surgery was performed through a median incision on the back, under chloral hydrate anesthesia. Two small cuts were made on the muscle $1-2 \mathrm{~cm}$ away from lumbar vertebrae 1 and 2 . The arteries beside the ovaries and the ovaries themselves were ligated and removed.

MCAO. A week after ovariectomy, the MCAO/control and $\mathrm{MCAO}+\mathrm{CMS}$ group rats were anesthetized with chloral hydrate $(3.2 \mathrm{mg} / \mathrm{kg})$. MCAO was induced using the intraluminal suture occlusion technique (31) and rats were placed on a temperature-controlled heating pad throughout surgery. The left common, external and internal carotid arteries were exposed. The external carotid artery was ligated and cut off, and the middle cerebral artery was occluded by inserting a
3-0 suture from the basal section of the external carotid artery and advanced cranially into the internal carotid artery. The suture was inserted $\sim 18 \mathrm{~mm}$ into the internal carotid artery and was carefully withdrawn $1.5 \mathrm{~h}$ after MCAO onset. The neurological deficits were evaluated by Bederson test (32). All scores of rats used in the present study were $>1$. Rats included in the sham group underwent sham surgery, which involved the same procedure, but without inserting the suture.

CMS procedure. The rats from the sham/CMS and $\mathrm{MCAO}+\mathrm{CMS}$ groups were exposed to CMS procedures for a period of two consecutive weeks, beginning one week after MCAO. The weekly stress procedure consisted of i) food and water deprivation for $24 \mathrm{~h}$; ii) cage tilt $\left(40^{\circ}\right)$, in which the rats' cages were tilted for $24 \mathrm{~h}$; iii) soiled cages, in which the sawdust in the cages was kept wet and dirty with $200 \mathrm{ml}$ water in the sawdust and bedding to maintain a poor living environment for $24 \mathrm{~h}$; iv) day and night reversal, involving turning on the light at 8:00 p.m. and off at 8:00 a.m.; v) restraint stress, which involved binding the rats to a fixator for $2 \mathrm{~h}$ so that the rats could not run or turn their bodies over; vi) cage tremor, in which the cages were put on a horizontal agitator that tremored continuously for $40 \mathrm{~min}$; and vii) clamping of the rats' tails (3 $\mathrm{cm}$ from the end) using $3-\mathrm{cm}$ clips. Each rat was isolated throughout the CMS procedure. The control groups were maintained in suitable living conditions.

Drug administration. Following the CMS procedure, a MCAO/CMS (PSD) group and a ham/control (control) group of rats were injected subcutaneously with $10 \mu \mathrm{g}$ 17 $\beta$-estradiol (E2758; Sigma-Aldrich, St. Louis, MO, USA) in 0.1 ml soybean oil between 9:00 and 10:00 a.m. every day for two weeks. In another PSD group and control group, the vehicle was administered in the same manner.

\section{Behavioral tests}

Sucrose preference test. Each group of rats had free access to two bottles containing $1 \%$ sucrose solution on the first day and two bottles, one containing $1 \%$ sucrose solution and the other containing water, on the second day in order to adjust to this test. The rats were deprived of food and water for $23 \mathrm{~h}$ prior to the sucrose preference test, which was performed as follows: Rats were provided with two bottles, one containing $1 \%$ sucrose solution and the other containing water, beginning between 9:00 and 10:00 a.m. Data were collected by weighing the two bottles after $1 \mathrm{~h}$, in order for the comparative consumption of sucrose solution and water to be analyzed and a preference calculated using the formula: Preference $(\%)=$ Sucrose solution intake / total intake $\mathrm{x} 100$. The baseline sucrose preference test was performed prior to MCAO, and the sucrose preference was monitored every week during periods of establishment of the model and treatment.

Open field test. A quadrate box was used for this experiment. The box was $40 \mathrm{~cm}$ in height, $100 \mathrm{~cm}$ in length and $100 \mathrm{~cm}$ in width. The wall was black and the floor was divided into 25 squares. Rats were placed on the floor and the scores of the horizontal movement and vertical movement were recorded for $3 \mathrm{~min})$. The baseline open field test performance was recorded prior to MCAO, and locomotory activity during the open field test was monitored every week whilst establishing the model. 
Forced swimming test. The forced swimming test is a standard test used to screen compounds for antidepressant-like activity. Swim sessions were conducted by placing rats in glass cylinders $\left(45 \mathrm{~cm}\right.$ tall $\mathrm{x} 18 \mathrm{~cm}$ in diameter) containing $23-25^{\circ} \mathrm{C}$ water at a $30-\mathrm{cm}$ depth. All rats were placed in this cylinder individually, with no escape platform, for $15 \mathrm{~min}$ as an initial pre-test. The following day, each rat was placed in the cylinders for $5 \mathrm{~min}$. Rats were then removed from the cylinders and dried with paper towels. During the test period, the time of 2 behaviors were recorded: i) Immobility: A rat was judged to be immobile when it remained floating in the water without struggling and was only making slight movements necessary to keep its head above water; and ii) struggling: A rat was judged to be struggling when it was making active attempts to escape from the cylinder, including searching for the escape routes and diving. This test was carried out every week during the period of treatment.

Western blot analysis. Animals were sacrificed after 2 weeks of treatment. Rats were anesthetized and decapitated, and their brains were rapidly removed and placed on ice for immediate dissection of the required brain regions. Dissected tissue was frozen and stored at $-80^{\circ} \mathrm{C}$ until the next experiment.

The hippocampus was isolated and homogenized in cell lysis buffer containing phenylmethanesulfonyl fluoride (Beyotime, Shanghai, China). Proteins (100 $\mu \mathrm{g}$ total protein) from hippocampus extracts were separated on $12 \%$ sodium dodecyl sulfate-polyacrylamide gels (Beyotime); following this, proteins were transferred to a polyvinylidene difluoride membrane (Beyotime) using electroblotting. The membranes were incubated with a blocking solution comprising Tris-buffered saline (Beyotime) and $0.05 \%$ Tween-20 (Beyotime) containing 5\% skimmed milk for 2 h. Following blocking, the membranes were incubated with rabbit anti-BDNF polyclonal antibody (1:200; ab6201; Abcam, Cambridge, MA, USA) at $4^{\circ} \mathrm{C}$ for $18 \mathrm{~h}$, then the membranes were washed and incubated with HRP-labeled goat anti-rabbit secondary antibody for $1 \mathrm{~h}$ at room temperature and developed using enhanced chemiluminescence. The X-OMAT BT films (Kodak, Rochester, NY, USA) were scanned and densitometric analyses were performed using Quantity One (Bio-Rad, Berkeley, CA, USA). $\beta$-actin was used as an endogenous loading control.

Statistics. A repeated measures analysis of variance (ANOVA), with treatment and time as the 2 variables, was used to analyze the data arising from behavioral tests. Post hoc analyses for multiple comparisons were made using a least significant difference (LSD) test. Group differences were considered statistically significant at $\mathrm{P}<0.05$. The BDNF protein level was analyzed by one-way ANOVA. Furthermore, results from post hoc tests using LSD measures with $\mathrm{P}<0.05$ were considered to be statistically significant.

\section{Results}

\section{Establishment of the PSD animal model}

Sucrose preference test. A repeated measures ANOVA of sucrose preference level between groups during model establishment determined that consumption of sucrose decreased during

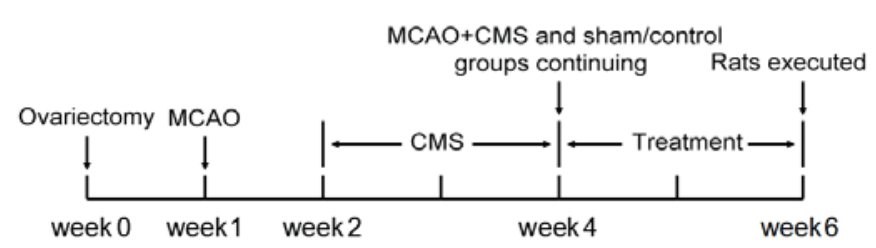

Figure 1. Schedule of the experiment, with a total duration of six weeks MCAO was induced one week after ovariectomy and the CMS procedure began one week after MCAO. CMS and drug treatment lasted two weeks each, prior to sacrifice. MCAO, middle cerebral artery occlusion; CMS, chronic mild stress

the CMS procedure. Variance (group vs. time) was analyzed by repeated measures, revealing no identifiable significant effects with regard to time $(\mathrm{F}=3.015 ; \mathrm{dF}=2 ; \mathrm{P}=0.055)$, but a significant difference among groups in terms of sucrose preference $\left(\mathrm{F}_{(3,76)}=12.85 ; \mathrm{P}<0.05\right)$. Sucrose preference was considerably reduced in the $\mathrm{MCAO}+\mathrm{CMS}$ group compared with the other groups, arising after only one week of stress and becoming markedly different on the second week (Fig. 2A); post hoc analysis revealed that the difference between the MCAO+CMS and sham/control groups was statistically significant $(\mathrm{P}<0.05)$. The sucrose preference of the MCAO+CMS group was lower than that of the sham/CMS group (Fig. 2A), but there was no statistical difference between the two groups. This result demonstrated severe depression in the MCAO+CMS group, meaning that MCAO+CMS treatment was successful in establishing a PSD model. Due to the destruction of brain tissue, the sucrose preference of the MCAO/control group declined, but the difference between the MCAO+CMS and $\mathrm{MCAO} /$ control groups was statistically significant $(\mathrm{P}<0.05)$ (Fig. 2A).

Open field test. In order to further demonstrate the severity of depression, rats were also assessed for changes in activity. Variance (group vs. time) analyses demonstrated a significant time-by-treatment interaction $(\mathrm{F}=2.322 ; \mathrm{dF}=6 ; \mathrm{P}=0.042)$. Rats gradually adapted to the open field box and groups undergoing CMS demonstrated significantly reduced horizontal $(\mathrm{P}<0.05$; Fig. 2B) and vertical activity $(\mathrm{P}<0.05$; Fig. 2C). An acute reduction in movement occurred in the first week of model establishment. A significant difference was reported between the groups after two weeks in terms of horizontal $\left(\mathrm{F}_{(3,76)}=9.586\right.$; $\mathrm{P}<0.05$; Fig. 2B) and vertical $\left(\mathrm{F}_{(3,76)}=7.929\right.$; $\mathrm{P}<0.05$; Fig. $\left.2 \mathrm{C}\right)$ movement. Post hoc analyses demonstrated a significant difference between the MCAO+CMS group and the other groups in terms of horizontal and vertical movement $(\mathrm{P}<0.05)$. The $\mathrm{MCAO}+\mathrm{CMS}$ rats were less curious to explore their surroundings and exhibited depression.

\section{Evaluation of the antidepressive effects of estrogen}

Sucrose preference test. The sucrose preference of the vehicle-treated and estrogen-treated groups was compared; two weeks of estrogen treatment led to a significant increase in sucrose preference in the E2-treated PSD groups $(\mathrm{P}<0.05)$, but no significant change in the vehicle-treated groups (Fig. 3A). The sucrose preference of the E2-treated PSD group was similar to the control groups, although a statistically significant difference remained.

Forced swimming test. Immobility time was reduced and duration of struggling increased in the E2-treated PSD 
group over the treatment period, when compared with the vehicle-treated PSD group $(\mathrm{P}<0.05)$, approaching the mean levels of the control groups. However, the difference in immobility between the E2-treated PSD group and the two control groups remained statistically significant (Fig. 3B). The struggle time of the E2-treated PSD rats significantly increased following E2 treatment $(\mathrm{P}<0.05$; Fig. $3 \mathrm{C})$; however, no significant difference was identified between the E2-treated PSD group and the two control groups ( $>0.05)$, indicating a rescue of mood in the PSD group following estrogen treatment

Expression of BDNF in the hippocampus. Protein level was measured by western blotting after two weeks of treatment. The BDNF band is indicated at $\sim 28 \mathrm{kDa}$. The ratio of BDNF: $\beta$-actin was used for statistical analysis. This ratio was different between the PSD groups $(\mathrm{P}=0.014)$, suggesting that the BDNF expression of the E2-treated PSD group was significantly increased compared with the vehicle-treated PSD group $(\mathrm{P}<0.05)$. No significant difference between the E2-treated PSD and control groups was identified $(\mathrm{P}>0.05$; Fig. 4).

\section{Discussion}

PSD occurs at high incidence rates in male and female stroke patients (1), but numerous previous studies have also revealed gender differences in PSD occurrence; these studies have predominantly reported a higher prevalence of depression among women than men (33). The higher frequency of PSD in female patients may be associated with medical conditions, likelihood of rehabilitation and hormonal changes, amongst other causes, but hormonal level is considered to be a particularly noteworthy contributory factor. Age is similarly correlated with the frequency of PSD (34); female stroke patients are typically of an advanced age (34) and commonly undergoing menopause. This indicates that changes to estrogen levels may be a key contributory factor to the high prevalence of PSD in female patients. As a consequence, ovariectomy-treated rats were selected for use in the present study.

In the current study, a PSD model was established through MCAO, followed by CMS, to imitate the pathogenesis of PSD. PSD rats demonstrated severe depressive behaviors compared with MCAO and CMS rats, based on sucrose preference and open field tests. Brain injury and poor living conditions in the present study may have contributed to the observed mood disorders; these were introduced to recapitulate clinical conditions, in which depression is caused by pathological changes to the brain and changes to lifestyle following a stroke. In the present study, MCAO was used to mimic trauma to the brain, specifically in the region of the brain controlling emotion, and CMS conditions were used to imitate the difficult life changes encountered following a stroke. Stroke patients often feel useless when they are unable to perform tasks that were previously easy and living conditions become uncomfortable due to their disability, so the present study aimed to replicate this.

The effects of estrogen on BDNF expression in the hippocampus of the PSD rats and on animal behavior, reflective
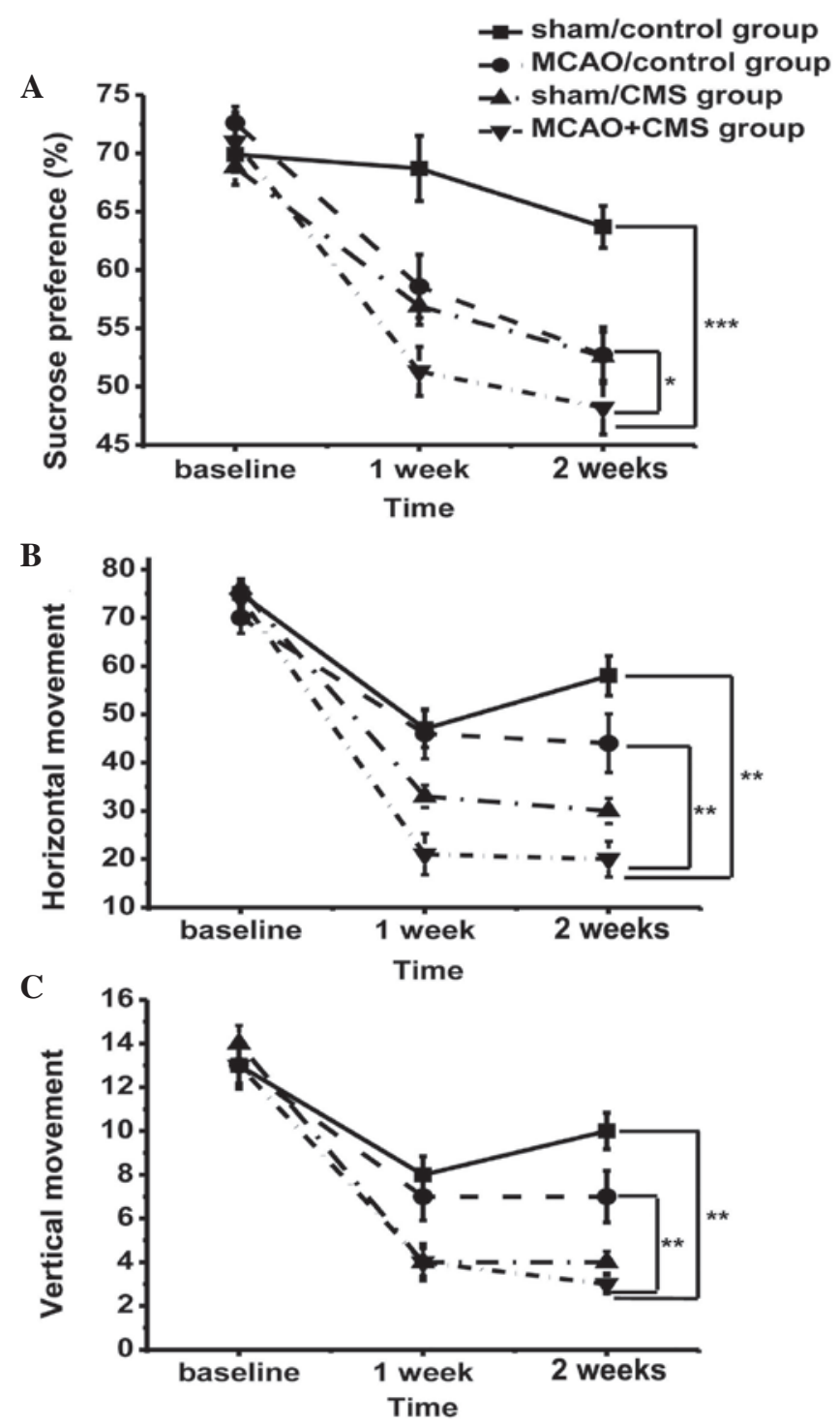

Figure 2. (A) Performance of rats in the sucrose preference test; and (B) horizontal and (C) vertical movement in the open field test prior to MCAO (baseline), and after one and two weeks, prior to the CMS procedure. ${ }^{*} \mathrm{P}<0.05 ;{ }^{* *} \mathrm{P}<0.01$. MCAO, middle cerebral artery occlusion; CMS, chronic mild stress.

of mood, were analyzed. Estrogen replacement rescued BDNF protein expression in the hippocampus and improved depression following a stroke. Preference of sucrose and performance in a forced swimming test were regarded as key indicators to evaluate the antidepressant effect during treatment. The key finding of the present study was that estrogen could significantly improve depression in PSD rats, according to these metrics, through regulation of BDNF.

Estrogen has been revealed to improve depression and to have neuroprotective effects on stroke patients (35). The present study demonstrated the therapeutic action of estrogen on the bio-psycho-social onset of PSD. Previous studies have reported that estrogen regulates the cell cycle at the G1/S transition, boosts neurogenesis following ER activation (36), and promotes B-cell lymphoma 2 expression and a decrease in apoptosis $(37,38)$. The inflammatory reaction following stroke causes expansive damage in the brain (39), 


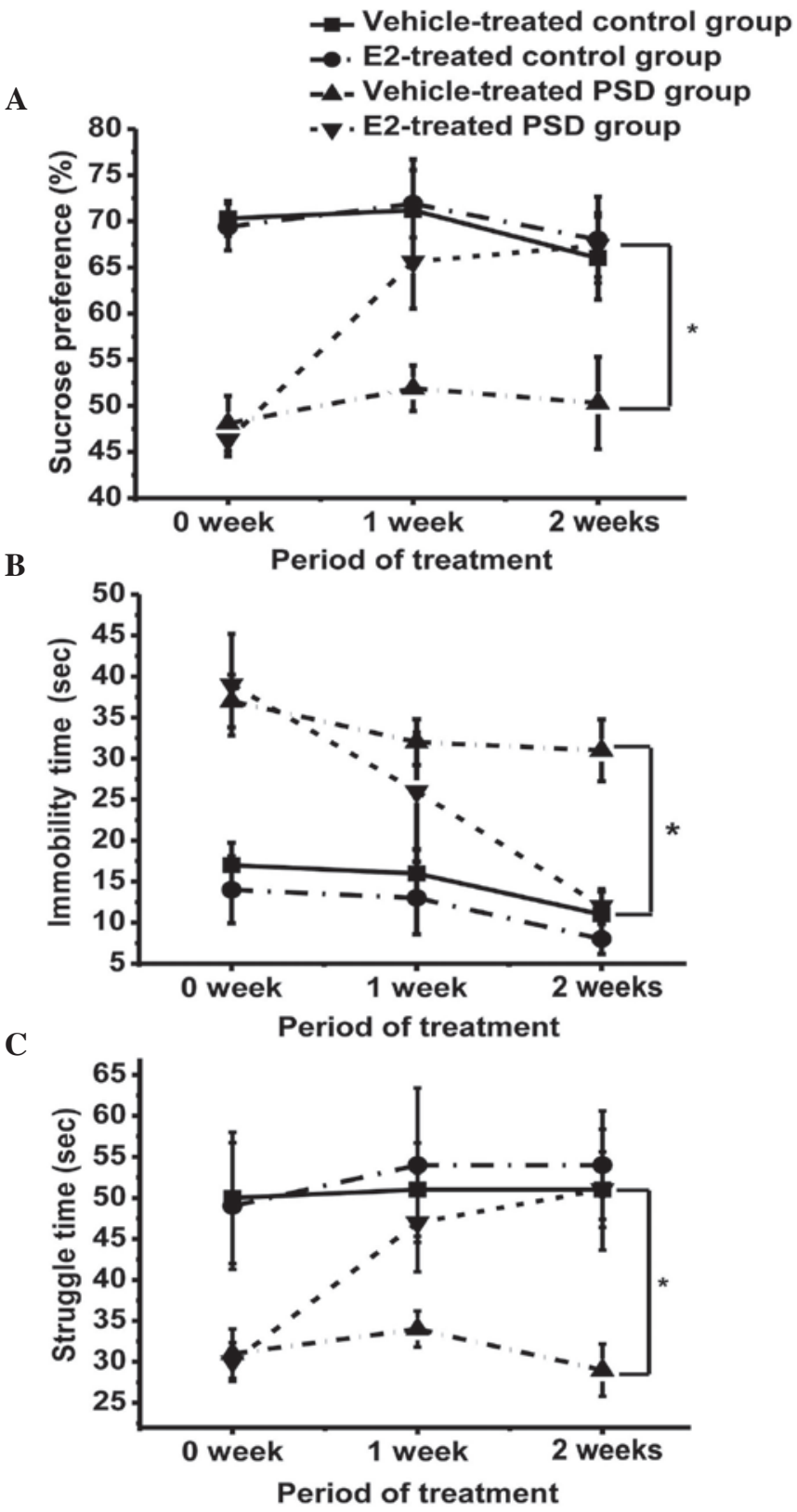

Figure 3. Effect of E2 on (A) sucrose preference, (B) time spent immobile during the forced swimming test and (C) struggle time in the forced swimming test. Values are presented as the mean \pm standard error of the mean. ${ }^{*} \mathrm{P}<0.05$. E2, 17 $\beta$-estradiol; PSD, post-stroke depression.

but this secondary injury following stroke can be blocked by estrogen (35). Consequently, neurological function is significantly improved following estrogen treatment due to increased neurogenesis and decreased ischemia-associated apoptosis (35). Estrogen may thus be an appropriate treatment for PSD, as confirmed by the present study.

BDNF has an important function in emotional stability; it has been associated with the development of depression in clinical and experimental studies (40), and is an important marker to evaluate the severity of depression. In a previous study, the BDNF level was low in PSD patients (21), and the present study reiterated this finding, demonstrating that BDNF expression in the PSD rats was substantially downregulated. In the current study, BDNF expression was
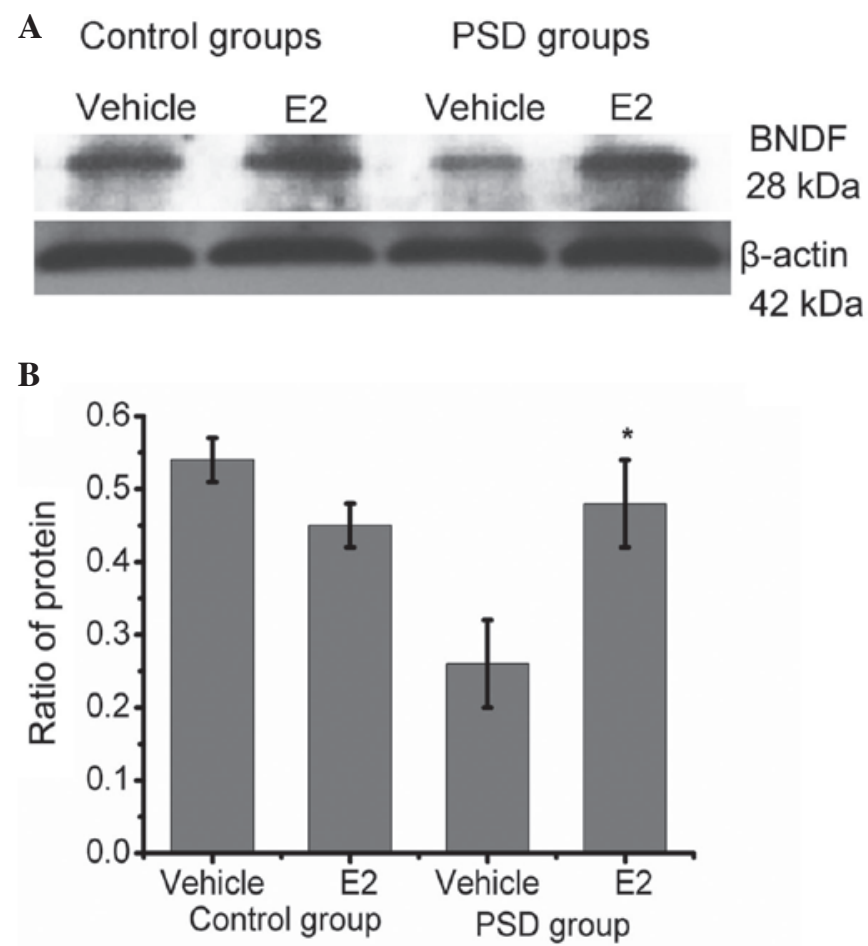

Figure 4. Western blot analysis of hippocampal BDNF expression in the E2- and vehicle-treated control groups, and the E2- and vehicle-treated PSD groups. (A) Representative image of western blotting. The BDNF and $\beta$-actin bands were observed at $28 \mathrm{kDa}$ and $42 \mathrm{kDa}$, respectively. Values are presented as the mean \pm standard error of the mean. (B) Histogram showing the ratio of protein. BDNF expression was upregulated in the PSD group following E2 treatment. ${ }^{*} \mathrm{P}=0.01$ when compared with the ratio of the proteins after treatment with the vehicle. No significant difference was identified between the E2-treated PSD group and the two control groups. BDNF, brain-derived neurotrophic factor; E2, 17ß-estradiol; PSD, post-stroke depression.

significantly increased in the PSD group treated with E2, approaching the BDNF level of the control group.

There are a number of similarities between estrogen and BDNF, which interact to exert their effects $(41,42)$. A possible hypothesis is that estrogen can affect BDNF expression and that estrogen can promote neurogenesis. ER activation enhances cell proliferation (36), which increased BDNF expression following estrogen treatment in the present study. Taken together, it appears plausible that estrogen promotes the release of BDNF, subsequently regulating the depressive mood following a stroke.

The estrogen-BDNF interaction begins an important molecular cascade to modulate the function of the hippocampus, which may be responsible for the antidepressant effects associated with estrogen treatment.

In summary, estrogen replacement therapy is suitable for the treatment of the PSD observed in ovariectomized rats and BDNF signaling may be the critical pathway by which estrogen is able to treat PSD; these findings may contribute to the treatment of PSD patients.

\section{Acknowledgements}

The present study was supported by grants from Wenzhou Technology Division (no. H20090067) and the National Institute of Health (nos. AG21980 and NS057186). 


\section{References}

1. Hackett ML, Yapa C, Parag V and Anderson CS: Frequency of depression after stroke: A systematic review of observational studies. Stroke 36: 1330-1340, 2005.

2. Weimar C, Kurth T, Kraywinkel K, Wagner M, Busse O, Haberl RL and Diener HC; German Stroke Data Bank Collaborators: Assessment of functioning and disability after ischemic stroke. Stroke 33: 2053-2059, 2002.

3. Williams LS, Ghose SS and Swindle RW: Depression and other mental health diagnoses increase mortality risk after ischemic stroke. Am J Psychiatry 161: 1090-1095, 2004.

4. Sinyor D, Amato P, Kaloupek DG, Becker R, Goldenberg M and Coopersmith H: Post-stroke depression: Relationships to functional impairment, coping strategies, and rehabilitation outcome Stroke 17: 1102-1107, 1986.

5. Carod-Artal FJ and Egido JA: Quality of life after stroke: The importance of a good recovery. Cerebrovasc Dis 27 (Suppl 1): 204-214, 2009.

6. Feibel JH and Springer CJ: Depression and failure to resume social activities after stroke. Arch Phys Med Rehabil 63: 276-277, 1982.

7. Janzing JG, Bouwens JM, Teunisse RJ, Van't Hof MA and Zitman FG: The relationship between depression and mortality in elderly subjects with less severe dementia. Psychol Med 29: 979-983, 1999.

8. Bilge C, Koçer E, Koçer A and Türk Börü U: Depression and functional outcome after stroke: The effect of antidepressant therapy on functional recovery. Eur J Phys Rehabil Med 44: 13-18, 2008.

9. Rossi C, Angelucci A, Costantin L, Braschi C, Mazzantini M, Babbini F, Fabbri ME, Tessarollo L, Maffei L, Berardi N and Caleo M: Brain-derived neurotrophic factor (BDNF) is required for the enhancement of hippocampal neurogenesis following environmental enrichment. Eur J Neurosci 24: 1850-1856, 2006.

10. Peng $\mathrm{CH}$, Chiou SH, Chen SJ, Chou YC, Ku HH, Cheng CK, Yen CJ, Tsai TH, Chang YL and Kao CL: Neuroprotection by Imipramine against lipopolysaccharide-induced apoptosis in hippocampus-derived neural stem cells mediated by activation of BDNF and the MAPK pathway. Eur Neuropsychopharmacol 18: 128-140, 2008

11. Luellen BA, Bianco LE, Schneider LM and Andrews AM: Reduced brain-derived neurotrophic factor is associated with a loss of serotonergic innervation in the hippocampus of aging mice. Genes Brain Behav 6: 482-490, 2007.

12. Hall J, Thomas KL and Everitt BJ: Rapid and selective induction of BDNF expression in the hippocampus during contextual learning. Nat Neurosci 3: 533-535, 2000

13. Duman RS and Monteggia LM: A neurotrophic model for stress-related mood disorders. Biol Psychiatry 59: 1116-1127, 2006

14. Mohajerani MH, Sivakumaran S, Zacchi P, Aguilera P and Cherubini E: Correlated network activity enhances synaptic efficacy via BDNF and the ERK pathway at immature CA3 CA1 connections in the hippocampus. Proc Natl Acad Sci USA 104: 13176-13181, 2007.

15. Li Y, Luikart BW, Birnbaum S, Chen J, Kwon CH, Kernie SG, Bassel-Duby R and Parada LF: TrkB regulates hippocampal neurogenesis and governs sensitivity to antidepressive treatment. Neuron 59: 399-412, 2008.

16. Bergami M, Rimondini R, Santi S, Blum R, Götz M and Canossa M: Deletion of TrkB in adult progenitors alters newborn neuron integration into hippocampal circuits and increases anxiety-like behavior. Proc Natl Acad Sci USA 105: 15570-15575, 2008.

17. Siuciak JA, Lewis DR, Wiegand SJ and Lindsay RM: Antidepressant-like effect of brain-derived neurotrophic factor (BDNF). Pharmacol Biochem Behav 56: 131-137, 1997.

18. Sen S, Duman R and Sanacora G: Serum brain-derived neurotrophic factor, depression, and antidepressant medications: Meta-analyses and implications. Biol Psychiatry 64: 527-532, 2008.

19. Satomura E, Baba H, Nakano Y, Maeshima H, Suzuki T and Arai $\mathrm{H}$ : Correlations between brain-derived neurotrophic factor and clinical symptoms in medicated patients with major depression. J Affect Disord 135: 332-335, 2011.

20. Dreimüller N, Schlicht KF, Wagner S, Peetz D, Borysenko L, Hiemke C, Lieb K and Tadić A: Early reactions of brain-derived neurotrophic factor in plasma (pBDNF) and outcome to acute antidepressant treatment in patients with Major Depression. Neuropharmacology 62: 264-269, 2012.

21. Yang L, Zhang Z, Sun D, Xu Z, Yuan Y, Zhang X and Li L: Low serum BDNF may indicate the development of PSD in patients with acute ischemic stroke. Int J Geriatr Psychiatry 26: 495-502, 2011.
22. Allaman I, Papp M, Kraftsik R, Fiumelli H, Magistretti PJ and Martin JL: Expression of brain-derived neurotrophic factor is not modulated by chronic mild stress in the rat hippocampus and amygdala. Pharmacol Rep 60: 1001-1007, 2008.

23. Romano-Torres M and Fernández-Guasti A: Estradiol valerate elicits antidepressant-like effects in middle-aged female rats under chronic mild stress. Behav Pharmacol 21: 104-111, 2010.

24. Herrick SP, Waters EM, Drake CT, McEwen BS and Milner TA: Extranuclear estrogen receptor beta immunoreactivity is on doublecortin-containing cells in the adult and neonatal rat dentate gyrus. Brain Res 1121: 46-58, 2006.

25. Walf AA, Rhodes ME and Frye CA: Antidepressant effects of ERbeta-selective estrogen receptor modulators in the forced swim test. Pharmacol Biochem Behav 78: 523-529, 2004.

26. Tanapat P, Hastings NB, Reeves AJ and Gould E: Estrogen stimulates a transient increase in the number of new neurons in the dentate gyrus of the adult female rat. J Neurosci 19: 5792-5801, 1999.

27. Crispino M, Stone DJ, Wei M, Anderson CP, Tocco G, Finch CE and Baudry M: Variations of synaptotagmin I, synaptotagmin IV, and synaptophysin mRNA levels in rat hippocampus during the estrous cycle. Exp Neurol 159: 574-583, 1999.

28. Li XH, Liu NB, Zhang MH, Zhou YL, Liao JW, Liu XQ and Chen HW: Effects of chronic multiple stress on learning and memory and the expression of Fyn, BDNF, TrkB in the hippocampus of rats. Chin Med J (Engl) 120: 669-674, 2007.

29. Sandstrom NJ and Williams CL: Spatial memory retention is enhanced by acute and continuous estradiol replacement. Horm Behav 45: 128-135, 2004.

30. Liu F, Day M, Muñiz LC, Bitran D, Arias R, Revilla-Sanchez R, Grauer S, Zhang G, Kelley C, Pulito V, et al: Activation of estrogen receptor-beta regulates hippocampal synaptic plasticity and improves memory. Nat Neurosci 11: 334-343, 2008.

31. Zhao H, Mayhan WG and Sun H: A modified suture technique produces consistent cerebral infarction in rats. Brain Res 1246: 158-166, 2008.

32. Bederson JB, Pitts LH, Tsuji M, Nishimura MC, Davis RL and Bartkowski H: Rat middle cerebral artery occlusion: Evaluation of the model and development of a neurologic examination. Stroke 17: 472-476, 1986.

33. Herrmann N, Black SE, Lawrence J, Szekely C and Szalai JP: The Sunnybrook Stroke Study: A prospective study of depressive symptoms and functional outcome. Stroke 29: 618-624, 1998.

34. Berg A, Palomäki H, Lehtihalmes M, Lönnqvist J and Kaste M: Poststroke depression: An 18-month follow-up. Stroke 34: 138-143, 2003.

35. Suzuki S, Brown CM and Wise PM: Neuroprotective effects of estrogens following ischemic stroke. Front Neuroendocrinol 30: 201-211, 2009.

36. Mazzucco CA, Lieblich SE, Bingham BI, Williamson MA, Viau V and Galea LA: Both estrogen receptor alpha and estrogen receptor beta agonists enhance cell proliferation in the dentate gyrus of adult female rats. Neuroscience 141: 1793-1800, 2006

37. Alkayed NJ, McCune SK, Crain BJ, Traystman RJ and Hurn PD: Estrogen-enhanced expression of Bcl-2 mRNA in rat brain after experimental stroke. FASEB J 12: A954, 1998.

38. Bagetta G, Chiappetta O, Amantea D, Iannone M, Rotiroti D, Costa A, Nappi G and Corasaniti MT: Estradiol reduces cytochrome $\mathrm{c}$ translocation and minimizes hippocampal damage caused by transient global ischemia in rat. Neurosci Lett 368: 87-91, 2004.

39. Danton GH and Dietrich WD: Inflammatory mechanisms after ischemia and stroke. J Neuropathol Exp Neurol 62: 127-136, 2003.

40. Sairanen M, Lucas G, Ernfors P, Castrén M and Castrén E: Brain-derived neurotrophic factor and antidepressant drugs have different but coordinated effects on neuronal turnover, proliferation, and survival in the adult dentate gyrus. J Neurosci 25: 1089-1094, 2005.

41. Zhou J, Zhang H, Cohen RS and Pandey SC: Effects of estrogen treatment on expression of brain-derived neurotrophic factor and cAMP response element-binding protein expression and phosphorylation in rat amygdaloid and hippocampal structures. Neuroendocrinology 81: 294-310, 2005.

42. Yang LC, Zhang QG, Zhou CF, Yang F, Zhang YD, Wang RM and Brann DW: Extranuclear estrogen receptors mediate the neuroprotective effects of estrogen in the rat hippocampus. PLoS One 5: e9851, 2010. 